Ann. Biol. anim. Bioch. Biophys., I973, 13 (2), 307-3II.

\title{
EFFET DE L'ABLATION DU MUSCLE SEMITENDINOSUS SUR LES MUSCLES ADJACENTS CHEZ BOS TAURUS
}

\author{
O. SCHMITT et B.-L. DUMONT \\ Laboratoire de Recherches sur la Viande, \\ Centre national de Recherches zootechniques, I. N.R. A., \\ 78350 Jouy en Josas
}

Dans le corps chaque muscle est une unité spécifique du système très complexe et bien équilibré que représente l'ensemble de la musculature. Chacun des muscles joue un rôle particulier, par exemple dans la locomotion, le maintien de la posture ou le mouvement des parois thoraciques ou abdominales. On peut donc supposer que l'absence d'un muscle déterminé pertuberait dans une certaine mesure la région anatomique où il accomplit son action.

L'absence de différents muscles chez l'Homme a été décrite par divers auteurs depuis de nombreuses années (LE Double, I897; BING, 1902). On considère qu'il s'agit là principalement d'un défaut héréditaire dont les conséquences sont plus ou moins sévères selon la fonction du muscle manquant. Chez les bovins on ne connaît qu'un nombre restreint de désordres héréditaires affectant la musculature et qui concernent spécialement soit l'hypertrophie des animaux culs-depoulain, soit une dystrophie consécutive à l'atrophie du système nerveux (LAUVERGNe, 1968). A notre connaissance l'absence d'aucun muscle n'a pas encore été rapportée dans le cas de l'espèce bovine, de sorte que l'on ignore quels effets aurait l'absence d'un muscle donné sur le comportement de l'animal ainsi que sur la fonction ou le développement des muscles qui lui sont adjacents.

D'une façon générale l'influence de la croissance d'un muscle sur les autres muscles n'est pas bien connue. L'intérêt, dans ce domaine, s'est principalement porté sur l'incidence du degré de développement des muscles sur le squelette qui les supporte. En particulier chez le Rat on a étudié l'effet de l'ablation de muscles sur la variation de taille et de poids de divers os, comme le crâne ou le bassin (Ashton et Moore, r968).

Le travail préliminaire rapporté dans cette note a été entrepris pour apprécier dans quelle mesure les muscles adjacents sont affectés si l'on élimine l'un des principaux muscles de la cuisse, le muscle Semitendinosus, très précocement au cours du développement, par ablation quelques jours après la naissance.

\section{MATÉRIEL E'T MÉTHODES}

On a utilisé un veau femelle de race Française Frisonne Pie noire, pesant $43 \mathrm{~kg}$ et âgé de quatre jours. Après anesthésie au pentotal on a pratiqué en salle d'opération l'excision complète du muscle Semitendinosus du côté gauche. Après ligature de l'incision et injection de sérum antitétanique, le veau fut placé dans un box sur de la paille fraîche. 
L'animal fut tué à l'âge de quatorze mois. Son poids était alors de $364 \mathrm{~kg}$. Après éviscération, la carcasse fut fendue en deux moitiés le long de la colonne vertébrale et sur chacune des demicarcasses on préleva le membre postérieur. Ceux-ci (fig. I) furent pesés, photographiés et disséqués. Les muscles adjacents au muscle Semitendinosus furent coupés perpendiculairement à la direction générale des fibres, à l'endroit où la plus grande différence était observée dans l'aspect des deux cuisses. Les muscles sectionnés furent photographiés et on y préleva une tranche de deux centimètres d'épaisseur pour examens histologiques. Ces derniers furent pratiqués sur des sections, de $\mathrm{I}_{5}$ microns d'épaisseur, de la totalité des muscles selon la technique précédemment décrite (Schmitt et Dumont, r969).

\section{RÉSULTATS}

Cinq heures après l'opération le veau se levait et buvait un peu d'eau. Deux jours plus tard l'animal se comportait comme un sujet normal et se déplaçait sans attirer l'attention.

A l'âge d'un an la cicatrice de l'opération était complètement couverte de poils et seules quelques marques légères étaient apparentes sur le pelage dont la couleur noire atténuait les variations de relief. La différence de conformation entre les deux côtés était pratiquement inexistante. Même un juge de bétail entraîné aurait eu du mal à déceler une légère anomalie sur le côté gauche.

Le poids des deux demi-carcasses fut pratiquement le même $(96,8 \mathrm{~kg}$ pour le côté gauche et $97,2 \mathrm{~kg}$ pour le côté droit). Aucune différence ne fut constatée dans l'avant-main. La forme du quartier postérieur gauche était, par contre, légèrement différente de celle du droit, dont le bord postérieur dessinait un profil rectiligne alors que le bord du quartier postérieur gauche était concave. Avant dissection le quartier postérieur gauche présentait sur sa face interne une légère dépression, large mais peu profonde. Après enlèvement du gras sous-cutané, le côté gauche, où manquait le muscle Semitendinosus présentait un aspect curieux, comparé au côté droit " normal »: on y percevait l'existence d'une nette dépression à l'endroit où normalement le muscle Semitendinosus aurait dû se trouver. Toutefois la dépression était loin d'être suffisamment vaste pour y loger le muscle Semitendinosus normalement développé d'un animal de ce poids et de cet âge.

Après l'excision du muscle Semitendinosus du côté droit (le poids du muscle était de I 54I g) la comparaison simultanée des muscles adjacents des deux côtés offrait des différences intéressantes à rapporter. La différence la plus importante portait sur les bords postérieurs des muscles Biceps femoris ( $\left(\mathrm{A}^{\prime}\right)$ et Semimembranosus $\left(\mathrm{B}^{\prime}\right)$ du côté gauche. Ce phénomène est illustré à la figure 2 qui montre le contour réel des muscles considérés au niveau de la section de la partie inférieure (cf. fig. I) de chacun des muscles. Les bords étaient arrondis sur toute la longueur des muscles, mais l'arrondissement était très accentué à la partie inférieure de chacun des muscles. Les bords des muscles du côté droit (A et B) étaient, eux, normalement aigus.

D'autres observations furent faites sur les muscles disséqués. Le muscle Semimembranosus du côté gauche apparaissait, examiné dorsalement, plus long, moins large et moins lourd que celui du côté droit. Toutefois, quand on l'examinait sous sa face interne, son épaisseur était plus grande que celle du muscle du côté droit et il paraissait alors plus lourd que ce dernier.

Des observations identiques furent faites sur le muscle Biceps femoris du côté gauche et du côté droit. En fait le poids des muscles des deux côtés, gauche et droit, était semblable, et respectivement de 3 I I9 et 3214 g pour le muscle Semimembranosus et de 3520 g pour les deux muscles Biceps femoris.

L'aspect général des autres muscles était normal. Les os des deux cuisses ne montraient pas de différence de poids et de forme.

Les sections transversales dans la partie postéro-inférieure (cf. fig. I) des muscles Semitendinosus et Biceps femoris des deux côtés différaient de forme et de surface. L'observation la plus frappante était, du côté gauche, l'arrondissement des angles et la courbure du bord latéral des 


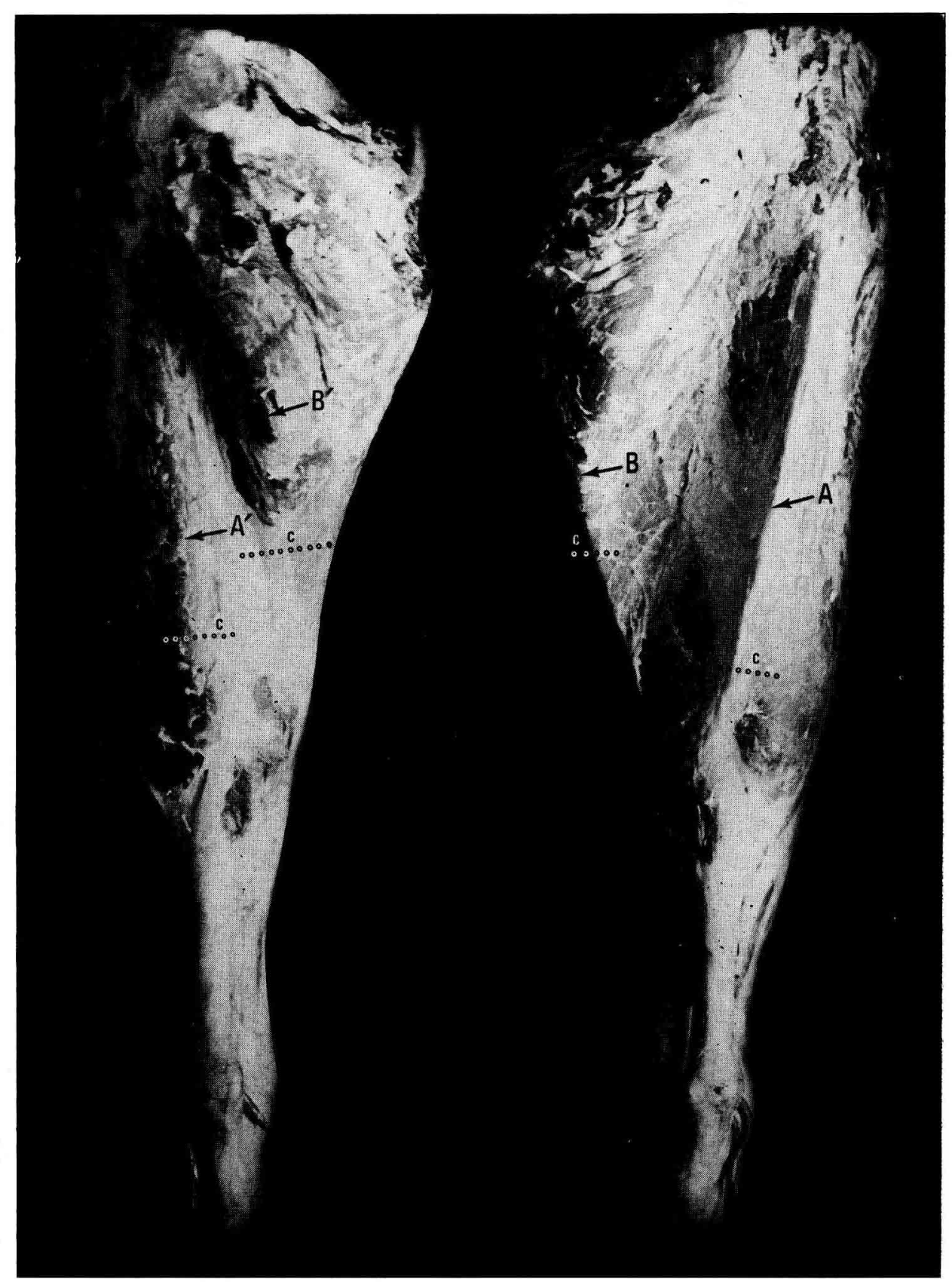

FIG. 1. - Face postérieure des cuisses gauche et droite de la génisse, après prélèvement, par dissection, du muscle Semitendinosus du côté droit

$\mathrm{A}^{\prime}$ : bord du muscle Biceps femoris gauche;

$\mathrm{B}^{\prime}$ : bord du muscle Semimembranosus gauche;

A : bord du muscle Biceps femoris droit;

B : bord du muscie Semimembranosus droit;

C : emplacement de la section transversale réalisée pour les études histologiques. 
muscles correspondant à l'espace où le muscle Semitendinosus était présent à la naissance. Dans le cas du Biceps femoris, la courbure s'accompagnait d'un agrandissement de la surface de section et d'un accroissement du diamètre transversal des fibres musculaires dans la partie élargie du muscle (la valeur moyenne du diamètre des fibres de cette partie, mesurée sur I 500 fibres, était de 68,6 microns alors qu'elle n'était que de 60,4 microns dans la région analogue du muscle droit)

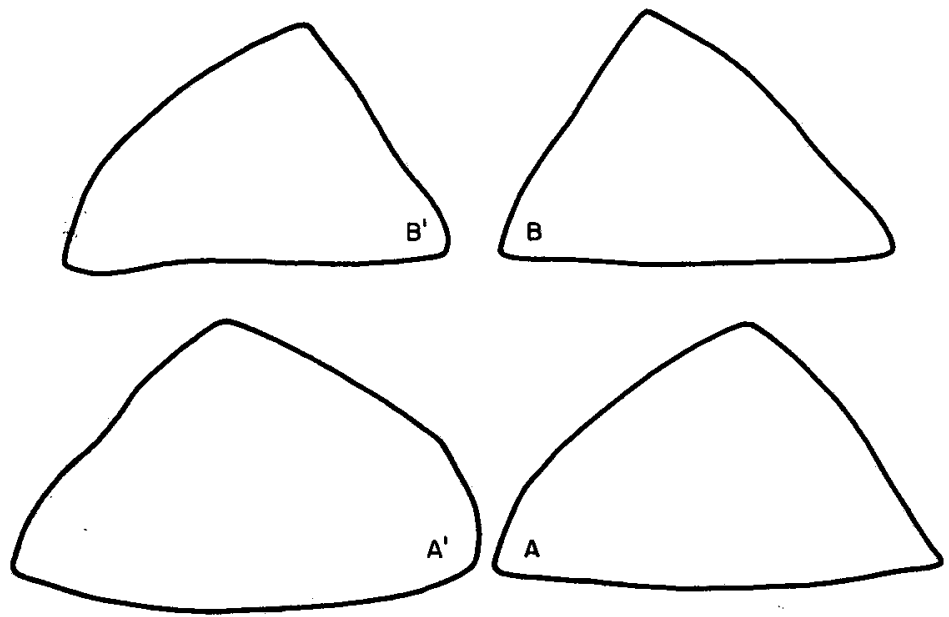

Fig. 2. - Contour des surfaces de section des muscles Semimembranosus en haut $(\times 0,25)$ et Biceps femoris en bas $(\times 0,5)$ au niveau c indiqué pour chacun d'eux à la figure_. 1

$A$ et $B$ bords postérieurs des muscles côté droit

$\mathrm{A}^{\prime}$ et $\mathbf{B}^{\prime}$ bords postérieurs des muscles côté gauche où a été pratiquée l'ablation du muscle Semitendinosus

\section{DISCUSSION}

La similitude des proportions des muscles des deux côtés doit être mise en parallèle avec le comportement de la génisse qui était tout à fait normal. L'absence du muscle Semitendinosus ne s'est accompagné, chez l'animal vivant, d'aucune modification notable de ses allures. A partir de ces observations le rôle du muscle Semitendinosus ne nous paraît pas clairement défini.

Les modifications de forme portent essentiellement sur le bord postérieur de chacun des muscles, dans une zone dont l'importance pondérale relative est faible eu égard à la masse totale du muscle, mais dont la situation rend particulièrement évidente les variations de contours qui peuvent s'y produire. D'autre part, il est établi qu'il existe une dissymétrie naturelle entre les poids des masses musculaires droites et gauches. Le caractère aléatoire de cette dissymétrie n'autorise pàs à prendre le poids d'un muscle d'un côté comme témoin de référence dans une étude d'un facteur expérimental affectant le muscle similaire de l'autre côté. Dans ces conditions une augmentation du volume de l'arête postérieure des muscles peut ne pas entraîner forcément un accroissement pondéral important. Quoi qu'il en soit on peut affirmer que les changements de forme des muscles Semimembranosus et Biceps femoris, à ce niveau, correspondent à un type d'hypertrophie.

Cette hypertrophie pourrait résulter de l'accroissement d'activité provoquée dans les muscles qui lui sont normalement adjacents par l'absence du muscle Semitendinosus. Cette hypothèse expliquerait une hypertrophie compensatrice du même type que celles qui ont été provoquées sur un muscle par différents auteurs, soit par ablation partielle de ce muscle ou par ténotomie de son synergique, comme cela a été fait chez la souris (Rowe et Goldspink, I968), chez le Rat 
(Hamosh et al., 1967) et le Poulet (Gutman et al., 1970). Il serait alors nécessaire de supposer que, chez les bovins, le Biceps femoris et le Semimembranosus peuvent en partie suppléer la déficience du muscle Semitendinosus. Cette hypothèse est en accord avec le comportement normal de la génisse pendant la marche ou la course.

Mais ce phénomène pourrait aussi être expliqué par la disparition, sur le côté gauche, de la contrainte physique provoqué, dans le membre postérieur normal, par la présence du muscle Semitendinosus. Avec une telle hypothèse il faut admettre que, chez un animal d'un âge ou d'un poids donné, les muscles sont normalement plus ou moins développés par rapport au potentiel génétique de croissance qu'ils avaient à la naissance, la cause principale de ce phénomène étant l'existence de forces contraires qu'exercent contre eux le développement des muscles qui les entourent ou d'autres causes mécaniques (par exemple la force de tension de la peau).

Reçu pour publication en janvier 1973.

\section{REMERCIEMENTS}

Nous sommes très reconnaissants au Dr P. Auffray d'avoir pratiqué l'ablation du muscle Semitendinosus. Nos remerciements vont aussi à M. PoNt pour les soins apportés à l'animal pendant sa période d'élevage et à $\mathrm{M}^{\mathrm{m}}$ e $\mathrm{DEGAS}$ pour sa participation au travail histologique.

\section{SUMMARY}

\section{EFFECT OF SEMITENDINOSUS ABLATION ON ADJACENT MUSCLES IN BOS TAURUS}

Ablation of the muscle Semitendinosus in a four-day old heifer resulted in modification of the form and area of the surrounding muscles (m. Semimembranosus and $\mathrm{m}$ Biceps femoris) fourteen months later. Concurrently, the size of muscular fibres is increased in the enlarged part of this muscle $(68.6 \mu$ versus $60.4 \mu)$. These results suggest that ablation of one muscle early in life may later induce a limited hypertrophy in the surrounding muscles. It is supposed that the muscles of intact animals do not realize their genetic growth potential because their development is limited by different types of physical restraint.

\section{RÉFÉRENCES BIBLIOGRAPHIQUES}

Ashton E. H., MOORE W. J., 1968. The effect of gluteal muscle ablation on the pelvis of the laboratory rat (Rattus norvegicus). J. Zool., London, 156, 155-16r.

Bing R., Igo2. Ueber angeborene Muskeldefecte. Virchows Arch. Pathol. Klin. Med. (Berlin), 170, I75-229.

Gutman E., HajeK I., VItek V., I97o. Compensatory hypertrophy of the Latissimus dorsi posterior muscle induced by elimination of the Latissimus dorsi anterior muscle of the chicken. Physiol., Bohemoslovaca, 19, 483-489.

Hamosh M., Lesch M., Baron J., Kaufman S., 1967. Enhanced protein synthesis in a cell-free system from hypertrophied skeletal muscle. Science, 157, 935-937.

LaUvergne J.-J., I968. Catalogue des anomalies héréditaires des bovins. Bulletin technique d'information du Département de Génétique animale, $\mathrm{n}^{\circ} \mathrm{r}$.

Le Double A.-F., I897. Traité des variations du système musculaire de l'Homme. Schleicher Frères, Éditeurs, Paris.

Rowe R. W. D., Goldspink G., I968. Surgically induced hypertrophy in skeletal muscles of the laboratory mouse. Anat. Rec., 161, 69-76.

Schmitt O., Dumont B.-L., r969. Méthodes d'analyse de la structure musculaire. Ann. Biol. anim. Bioch. Biophys., 9, 123-134. 